\title{
A filosofia da educação no Quebec: quais são os grandes nomes da educação no Quebec?
}

\section{Philosophy of education in Quebec: which are the great names of the education in the Quebec?}

\section{La philosophie de l'éducation au Québec: quels sont les grands noms de l'éducation au Québec?}

Stéphanie COTE

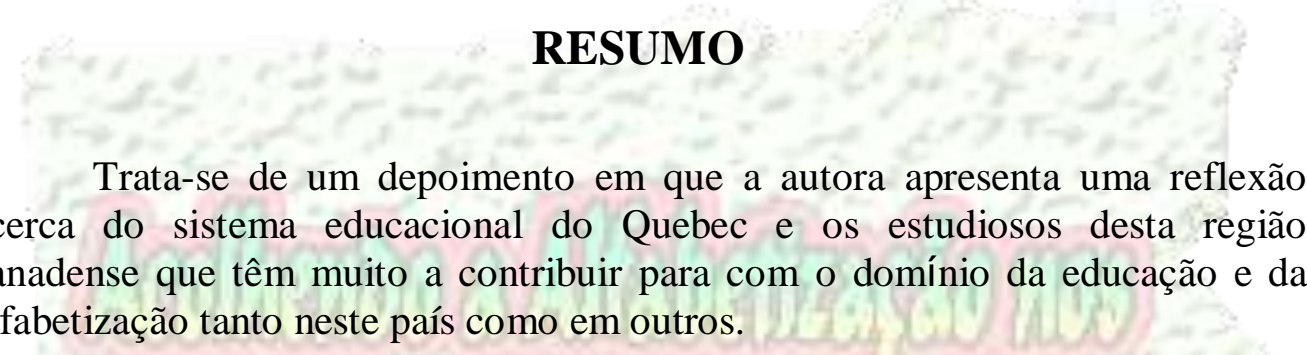

Palavras - chave: Quebec, estudiosos, contribuições, educação.

\section{ABSTRACT}

This report is a reflection concerning the Quebec educational system and studious persons of this region of Canada that have much to contribute with the education and literacy domain in their country as in others ones.

Index terms: Quebec, studious, contributions, education.

\section{RÉSUMÉ}

Il s'agit d'une temignage où l'auteure présente une réflexion concernant au système scolaire du Quebec et quelques studieux de cette région canadienne qui ont beaucoup d'à contribuer envers le domaine de l'éducation et de l'alphabétisation tant dans ce pays que dans d'autres.

Mots clés: Quebec, studieux, contributions, éducation. 
Cette temoignage fait ressortir quelques dates où il $\mathrm{y}$ a eu des changements, des rapports qui ont été écrits par le Ministère de l'Éducation du Québec, parmi lequels, j'ai trouvé une auteure, une chercheuse que j'aime beaucoup parce que ces travails peuvent aider beacoup la pratique dans le domaine de l'éducation, de l'orthopédagogie et aussi de l'alphabétisation. Alors, voici donc le fruit de mes recherches.

Tout d'abord, le Ministère de l'Éducation du Loisir et du Sport (MELS), a pour mission:

- de promouvoir l'éducation, le loisir et le sport;

- de contribuer, par la promotion, le développement et le soutien de ces domaines, à l'élévation du niveau scientifique, culturel et professionnel ainsi que du niveau de la pratique récréative et sportive de la population québécoise et des personnes qui la composent;

- de favoriser l'accès aux formes les plus élevées du savoir et de la culture à toute personne qui en a la volonté et l'aptitude;

- de contribuer à l'harmonisation des orientations et des activités avec l'ensemble des politiques gouvernementales et avec les besoins économiques, sociaux et culturels.

Dans cette optique, je pense que les personnes qui geuvrent pour le Ministère ont beaucoup de mérites face à tous les changements que nous avons en ce qui concerne l'éducation au Québec. Depuis sa création en 1964, le MELS tente, sans cesse, de faire évoluer l'éducation et être à la fine pointe afin de suivre la parade du $21^{\mathrm{e}}$ siècle. Pensons à l'évolution en ce qui a trait aux Technologies de l'Information et de la Communication (TICs) et à toute l'évolution suivie de la sortie du rapport COPEX sur les enfants en difficultés d'adaptation afin de leur permettre l'accessibilité à un service public, leur donner une éducation de qualité et assurer le droit aux EHDAA de grandir dans 
le cadre le plus normal possible. En plus, la Loi de l'instruction publique (LIP) est modifiée régulièrement afin de faire face à toutes les éventualités et aux changements d'aujourd'hui. D'ailleurs dans les années 80 , elle a été modifiée afin de favoriser davantage la participation parentale. Dans le même ordre d'idées, Micheline DESPRÉS-POIRIER a écrit le livre: «Le système d'éducation au Québec» $3^{\mathrm{e}}$ édition. Montréal, Gaëtan Morin. 1999 à ce sujet. En 2002, la Loi 101 sur la langue française a eu une incidence importante sur le système de l'éducation du Québec. Cette loi était simplement un moyen que nous nous donnions pour protéger ce que nous étions et sommes encore aujourd'hui : des Québécois (Éric Champagne). Dans les années 2000, il y a eu la sortie de la nouvelle Réforme. Même si cette dernière ne fait pas l'unanimité, nous n'avons pas le choix de la respecter et de préparer nos cours en la considérant. Les recherches avancent et tiennent également compte de la Réforme de l'éducation. Voilà les raisons pour lesquelles je crois et je pense sérieusement que les gens qui travaillent pour le Ministère sont des personnes, de grands noms, qui ont su faire évoluer le système de l'éducation au Québec.

Pour ce qui est de mon coup de cour, il s'agit de Mme. Line Laplante. C'est une professeure à l'Université du Québec à Montréal (UQÀM) au Département de linguistique et de didactique des langues pour la Faculté des sciences humaines. C'est également une chercheuse dans les domaines de spécialisation suivants: dyslexie, dysorthographie, lecture, écriture, démarche d'évaluation, intervention préventive, rééducation, acquisition normale lectureécriture et difficultés d'apprentissage. J'ai eu la chance d'assister à une de ses conférences données dans le cadre du colloque 2008 de «l'Association des Orthopédagogues du Québec (ADOQ)». Elle est très intéressante et connaît véritablement bien son sujet. Je ne crois pas qu'elle ait écrit un livre en particulier, mais lors des colloques, elle donne régulièrement des conférences 
par rapport à ses sujets de spécialisation. C'est une chercheuse active et très impliquée dans le domaine de l'adaptation scolaire. Je ne sais pas si c'est mon cœur d'orthopédagogue qui parle, mais c'est une personne que je respecte énormément et qui, à mon avis, a grandement fait évoluer le monde de l'éducation et des élèves en adaptation scolaire. J'aurais vraiment voulu donner une biographie plus complète d'elle mais je n'ai malheureusement pas trouvé plus de détails à son sujet.

Finalement, je considère que Jacqueline Caron est également une personne québécoise qui a fait évoluer le système de l'éducation du Québec. En fait, elle a écrit plusieurs ouvrages que nous avons eu la chance de survoler tout au long de ma formation en adaptation scolaire et sociale de 2004 à 2008. Elle a écrit: «Quand revient septembre...» Montréal: Éditions de la Chenelière, 19941997 - 2 volumes. Ces livres parlent de la gestion de la classe participative, des outils organisationnels et pratiques à utiliser, des activités à réaliser, etc. De plus, elle a écrit un livre sur la différenciation pédagogique: «Apprivoiser les différences: guide sur la différenciation des apprentissages et la gestion des cycles». C'est une auteure qui a le souci de la différenciation, de la gestion d'une classe et qui sait donner des moyens concrets et faciles à mettre en place.

En guise de conclusion, je dois dire qu'à mon avis, Jean Piaget a été un grand révolutionnaire de l'éducation au Québec. Par contre, comme il ne s'agit pas d'un homme québécois, je ne pouvais pas parler de lui. Ainsi, le Ministère, le Conseil supérieur de l'éducation et toutes les personnes qui faisaient des recherches ou qui ont travaillé pour eux, ont tout de même une part importante à tous ces changements d'aujourd'hui. Je lève mon chapeau à tous les gens qui font des recherches et qui ont le souci de l'évolution et du changement dans l'éducation. Il y a sans doute pleins d'autres personnes qui ont fait évoluer l'éducation du Québec, mais je me suis contentée de parler de seulement 
quelques-unes d'entre elles. Je me rends compte que j'ai parlé principalement de personnes qui ont fait des recherches dans le domaine de l'adaptation scolaire. Comme je suis une passionnée dans ce domaine, je réalise que ce travail reflète bien mes pensées. Même s'il n'a pas été très évident à faire, je dois admettre qu'il m'a permis de me questionner davantage et de me poser la question: «Quels sont les grands noms de l'éducation au Québec?»...

\section{Autora:}

Stéphanie Côté

Ortopedagoga. Atua em escolas na cidade de Chicoutimi, Quebec.

Contato: stephanie.cote@uqac.ca

Depoimento recebido em maio de 2008.

Depoimento aprovado para publicação em julho de 2008.

\section{Como citar este texto:}

CÔTÉ, S.. La phitosophie de T'éducation au Québec : quels sont les grands noms de l'éducation au Québec? Revista Acolhendo a Alfabetização nos Países de Língua Portuguesa, Brasil, São Paulo, volume 1, nº. 10, pp. 146 150, Mar. 2011. Disponível em: 〈http://www.acoalfaplp.net>. 